\title{
Implementing Outcomes-Based Managed Entry Agreements for Rare Disease Treatments: Nusinersen and Tisagenlecleucel
}

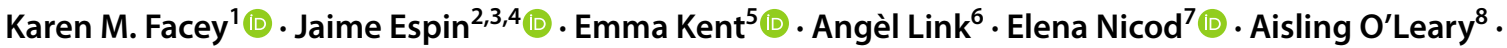 \\ Entela Xoxi ${ }^{9}$ (1) $\cdot$ Inneke van de Vijver ${ }^{10} \cdot$ Anna Zaremba $^{11} \cdot$ Tatyana Benisheva $^{12}$ (1) Andrius Vagoras ${ }^{13}$ (1) \\ Sheela Upadhyaya ${ }^{5}$
}

Accepted: 1 June 2021 / Published online: 7 July 2021

(c) The Author(s) 2021

\begin{abstract}
Background and Objective Enthusiasm for the use of outcomes-based managed entry agreements (OBMEAs) to manage uncertainties apparent at the time of appraisal/pricing and reimbursement of new medicines has waned over the past decade, as challenges in establishment, implementation and re-appraisal have been identified. With the recent advent of innovative treatments for rare diseases that have uncertainties in the clinical evidence base, but which could meet a high unmet need, there has been renewed interest in the potential of OBMEAs. The objective of this research was to review the implementation of OBMEAs for two case studies across countries in the European Union, Australia and Canada, to identify good practices that could inform development of tools to support implementation of OBMEAs.

Methods To investigate how OBMEAs are being implemented with rare disease treatments, we collected information from health technology assessment/payer experts in countries that had implemented OBMEAs for either nusinersen in spinal muscular atrophy or tisagenlecleucel in two cancer indications. Operational characteristics of the OBMEAs that were publicly available were documented. Then, the experts discussed issues in implementing these OBMEAs and specific approaches taken to overcome challenges.

Results The OBMEAs identified were based on individual outcomes to ensure appropriate use, manage continuation of treatment and in two cases linked to payment schedules, or they were population based, coverage with evidence development. For nusinersen, population-based OBMEAs are documented in Belgium, England and the Netherlands and individual-based schemes in Bulgaria, Ireland, Italy and Lithuania. For tisagenlecleucel, there were population-based schemes in Australia, Belgium, England and France and individual-based schemes in Italy and Spain. Comparison of the OBMEA constructs showed some clear published frameworks and clarity of the uncertainties to be addressed that were similar across countries. Agreements were generally made between the marketing authorisation holder and the payer with involvement of expert physicians. Only England and the Netherlands involved patients. Italy used its long-established, national, web-based, treatment-specific data collection system linked to reimbursement and Spain has just developed such a national treatment registry system. Other countries relied on a variety of data collection systems (including clinical registries) and administrative data. Durations of agreements varied for these treatments as did processes for interim reporting. The processes to ensure data quality, completeness and sufficiency for re-analysis after coverage with evidence development were not always clear, neither were analysis plans.

Conclusions These case studies have shown that important information about the constructs of OBMEAs for rare disease treatments are publicly available, and for some jurisdictions, interim reports of progress. Outcomes-based managed entry agreements can play an important role not only in reimbursement, but also in treatment optimisation. However, they are complex to implement and should be the exception and not the rule. More recent OBMEAs have developed document covenants among stakeholders or electronic systems to provide assurances about data sufficiency. For coverage with evidence development, there is an opportunity for greater collaboration among jurisdictions to share processes, develop common data collection agreements, and share interim and final reports. The establishment of an international public portal to host such reports would be particularly valuable for rare disease treatments.
\end{abstract}

Extended author information available on the last page of the article 


\section{Key Points for Decision Makers}

Outcomes-based managed entry agreements can in some circumstances help resolve uncertainties relating to the value and optimal use of a rare disease treatment, but they should be supported by a covenant agreed by all stakeholders providing assurance that all necessary actions will be taken to ensure that the data collected will be sufficient for decision making.

When used, outcomes-based managed entry agreement constructs for data collection and reports of clinical results should be published and shared to enable agreement of core data across jurisdictions, data aggregation and to support health system learnings.

An international public repository for outcomes-based managed entry agreement reports akin to the HTA database hosted by the International Network of Agencies for Health Technology Assessment would be valuable.

\section{Background}

Because of the difficulties associated with clinical research in rare diseases, treatments often come to market with a small evidence base involving uncontrolled or small randomised controlled trials of short duration, studying outcomes that may not clearly demonstrate patient benefit [1]. This leads to a range of uncertainties relating to the population appropriate for reimbursement, and to the determination of clinical and cost effectiveness. Alongside this, rare disease treatments are often associated with high prices, which challenge standard willingness-to-pay thresholds and raise concerns about budget impact, even after negotiation of financial/commercial agreements. Some countries have special appraisal and reimbursement mechanisms that allow flexibility in evaluation of rare disease treatments [2], such as leniency in critical assessment/review of evidence, flexibility in base-case assumptions for economic modelling and broader consideration of value. Despite these adapted approaches, some treatments for which substantial clinical benefit or large gains in quality-adjusted life-years are estimated are still associated with major uncertainties, which means they cannot be recommended for use or full reimbursement in a health system. Alongside this, the high unmet need, childhood onset and severity of many rare diseases means there is often strong clinical, patient/parent and political lobbying to provide access to such rare disease treatments. In these situations, one solution to access could be an outcomes-based managed entry agreement (OBMEA) ${ }^{1}$ that seeks to manage the clinical and financial uncertainties $[3,4]$.

Outcomes-based managed entry agreements can be used with any form of health technology and occur at two levels for a range of purposes including resolution of uncertainties in clinical evidence, management of budget impact, and improved understanding of real-life and long-term effectiveness to optimise care:

Individual: ensuring only eligible patients receive treatment, sometimes with assessment of outcomes to determine treatment continuation (appropriate use) or linked to payment schemes (paying only if response achieved or refund if response not achieved)

Population: using coverage with evidence development (CED)/post-licensing evidence generation as a conditional coverage mechanism post-health technology assessment (HTA)/reimbursement that requires evidence collection to inform re-appraisal or pricing and reimbursement re-negotiations

However, even with prevalent conditions, implementation of OBMEAs has been found to be challenging [5, 6] owing to administrative burdens on health providers/ payers and for population-based schemes, the insufficiency of data to inform re-appraisal and funding of data collection [7]. Pricing and reimbursement processes are the responsibility of the Ministry of Health nationally and/or a payer, which may have a "jurisdiction" that is national, relates to a region in the country or to a population covered by the health plan. Furthermore, these OBMEAs must be agreed alongside confidential commercial arrangements, thus the constructs of the OBMEAs are often kept confidential or published in local languages that have made sharing difficult. As a consequence, different agreements arise for the same treatment in different jurisdictions. This represents a challenge for the marketing authorisation holder (company/health technology sponsor), but for rare diseases this is also an important inefficiency as sharing of outcomes data across jurisdictions could create more robust evidence for re-appraisal $[8,9]$.

Work Package (WP) 10 of the European Commissionfunded H2020 IMPACT HTA project has undertaken research to develop an appraisal framework suitable for rare disease treatments that includes consideration of when and how to implement OBMEAs. For this, IMPACT HTA WP10 drew on the European Commission-funded H2020 COMED

\footnotetext{
${ }^{1}$ Including performance-based risk sharing schemes/agreements and coverage/post-licensing/access with evidence/data development/generation/collection.
} 
project. The COMED systematic review of CED schemes [10] identified a range of challenges, many of which seemed transferable to rare disease treatments. We categorised these into issues relating to:

- Establishment (including purpose, stakeholder responsibilities, agreement on eligibility and continuation criteria, outcomes to be studied)

- Implementation (including research protocol, data collection infrastructure, monitoring to improve data quality and completeness, amendment processes, feedback mechanisms for physicians and patients, resourcing, ethical issues)

- Re-appraisal (including ability to implement a revised reimbursement decision).

Reckers-Droog et al. [10] suggested that future research should seek to deepen understanding of the challenges of OBMEAs for different health technologies and consider impacts for all stakeholders including clinicians and patients. Hence, IMPACT HTA WP10 organised workshops with all stakeholders involved in National Institute for Health and Care Excellence (NICE) Highly Specialised Technologies (HST) OBMEAs (called managed access agreements). These OBMEAs gather data from a variety of health system sources for treatments of ultra-rare conditions that are provided in a national specialised service in NHS England. Then EX undertook a desktop review of the national webbased registry system of the Italian Medicines Agency, AIFA [11]. This in-depth evaluation of all AIFA registries over a 15-year period identified 88 registries for rare disease indications and explored their form, evolution and impact.

This work from England and Italy showed some differences in approaches to OBMEAs, but with a commonality of issues in relation to clinician (physicians and pharmacists) burden of data entry and assessment. As a result, it was decided to review the implementation of OBMEAs for two specific rare disease treatments across a much broader range of countries. The purpose was to explore how different health systems had established and implemented both individual and population-based OBMEAs and identify good practices that could inform development of tools to support OBMEAs for rare disease treatments.

\section{Methods}

Given emerging findings in other parts of IMPACT HTA WP10 research, two different treatments designated as orphan medicinal products (OMP) by the European Medicines Agency were chosen as case studies: nusinersen and tisagenlecleucel. Nusinersen (Spinraza ${ }^{\circledR}$ ) has European Union regulatory authorisation for $5 \mathrm{q}$ spinal muscular atrophy (SMA) and is administered as an intrathecal injection with four loading doses in the first 2 months and then a maintenance dose every 4 months thereafter, continued dependent on response. Tisagenlecleucel $\left(\mathrm{Kymriah}^{\circledR}\right)$ is a one-off CAR-T therapy with European Union regulatory authorisation for patients aged up to 25 years with refractory/relapsed B-cell acute lymphoblastic leukaemia or adults with relapsed/refractory diffuse large B-cell lymphoma. These provide contrasting cases of: non-cancer vs cancer; ongoing vs one-off treatment; babies/adults vs adolescents/ adults; antisense oligonucleotide vs advanced therapeutic medicinal products; different HTA/reimbursement routes within organisations.

With a good understanding of the processes to undertake OBMEAs at NICE and AIFA, a template was developed to capture relevant information about OBMEAs in other jurisdictions. In February 2020, the template was sent to HTA/payer/academic experts in each country in the European Union, European Economic Area, Canada, Australia and New Zealand that we had worked with in the documentation of appraisal processes in another workstream of IMPACT HTA WP10. They were asked to complete the templates using publicly available information. We sent several reminder e-mails over the coming months, but this coincided with the emergence of the COVID-19 pandemic and thus we were sensitive to health system pressures. Therefore, we contacted a market access expert from each marketing authorisation holder to identify the countries where their products had been reimbursed and those that were likely to have an OBMEA and targeted repeated follow-ups to these countries.

The completed templates were reviewed by KF to clarify entries and reduce duplication. Each revised table was validated by the relevant HTA expert. The HTA/payer/academic experts (authors) that contributed detailed information were invited to two virtual workshops in autumn 2020 to discuss their entries in the revised tables, differences in processes, new initiatives that had been implemented to overcome previous challenges with OBMEAs and the potential for future collaboration in relation to OBMEAs for rare disease treatments.

\section{Results}

\subsection{Data Returns}

\subsubsection{Nusinersen}

In the data collection phase in summer 2020, it was recognised that the following countries had not authorised/reimbursed nusinersen or were unlikely to have an OBMEA: Croatia, Denmark, Estonia, Greece, Iceland, Liechtenstein, Luxembourg, Malta, New Zealand, Romania and Slovakia 
and their non-response was not pursued beyond one followup. Canada, Finland, Scotland, Sweden and Switzerland did not respond. In Scotland, nusinersen is reimbursed for SMA type 1 and from July 2019 it could be prescribed for SMA types $2 / 3$ via the new ultra-orphan pathway. This agreement lasts for a period of up to 3 years while further evidence on effectiveness is generated, but no other details are available ${ }^{2}$. Canada, Sweden and Finland have decentralised systems, where the provinces/hospitals/councils negotiate pricing and reimbursement and any OBMEA, but these agreements are confidential.

For Australia, France, Germany, Hungary, Norway and Slovenia, we were informed that nusinersen was reimbursed, often with strict inclusion criteria, but without an OBMEA. According to the marketing authorisation holder, the Czech Republic does have a scheme that assesses effectiveness in children after 1 year of treatment and outcomes-based continuation criteria are applied, but no further details are available.

Templates returned from Austria, Portugal and Spain provided general responses about OBMEA construction and had few details for the specific treatment because the agreements are confidential. Austria made regional reimbursement decisions on nusinersen including for indications and any associated OBMEA, but it is known that one region collected motor milestones in the German SMArtCare registry ${ }^{3}$.

For the remaining countries, templates were returned showing CED (population-based) schemes in Belgium, England and the Netherlands (Table 1). Responses from Bulgaria, Ireland, Italy and Lithuania showed individualbased OBMEAs used to determine eligibility, continuation and, in some cases, total budget caps that are intended to be used in pricing and reimbursement renegotiations (Table 2).

An initial response was received from Latvia that documented an individual-based scheme, but because of health system pressures, it has not been possible to validate the data summary. In Poland, it is unclear if the scheme proposed by the HTA body was implemented. These are shown in Table 2 in italic to enable comparisons to the individualbased schemes that were implemented, and for which there is validated information.

\subsubsection{Tisagenlecleucel}

In the data collection phase, it was determined from respondents or the marketing authorisation holder that at that point in time, Bulgaria, Estonia, Hungary, Ireland, Latvia, Lithuania, Slovakia and Slovenia had not authorised/ reimbursed tisagenlecleucel and that Croatia, Cyprus, Denmark, Greece, Iceland, Liechtenstein, Luxembourg, Malta,

\footnotetext{
${ }^{2}$ Nusinersen (Spinraza) (scottishmedicines.org.uk).

${ }^{3}$ https://www.smartcare.de/en/.
}

New Zealand and Romania did not have OBMEAs. Contacts in the Czech Republic, Finland and Switzerland did not respond. For Portugal, a general response about the process was received indicating that any agreement would be made with INFARMED and the marketing authorisation holder, but that there was a preference for simple discounts.

Norway, Scotland and Sweden had reimbursed tisagenlecleucel through standard appraisal and reimbursement processes without need for an OBMEA. The Netherlands only uses population-based OBMEA for non-cancer OMPs, but can establish an individual-based agreement to ensure appropriate use. It did not use this for tisagenlecleucel, but gave reimbursement for acute lymphoblastic leukaemia only. In Poland, there were originally applications for emergency access for a limited group of patients, then applications for reimbursement were made.

Jørgensen et al. [12] stated that in Germany during the initial 12-month free-pricing period, the marketing authorisation holder made arrangements with the health insurers, covering $60 \%$ of the eligible population, for an outcomesbased rebate for patients who died within a certain period. After the early benefit assessment, the reimbursement mechanism has reverted to a simple confidential discount.

In Austria, for tisagenlecleucel, there was a national agreement on the reimbursed indication agreed by the national specialist group of haematologists ${ }^{4}$ and outcomes data are documented in the European Society for Blood and Marrow Transplantation. Reimbursement decisions (including any OBMEA) were agreed in regions and are confidential.

In Canada, CADTH recommended that tisagenlecleucel be provided via interprovincial agreements with clear eligibility criteria. It was recommended that standardised outcomes data be collected in a pan-Canadian registry to generate real-world evidence for consideration in future assessments to assess longer term effectiveness, safety and cost effectiveness ${ }^{5}$. No further details are published.

Templates were returned showing CED schemes in Australia, Belgium and England and an individual-based scheme in Italy. Publications and public reimbursement information described CED in France and an individual-based scheme in Spain. These are summarised in Table 3.

\subsection{Country Comparisons Reimplementing OBMEAs}

Every jurisdiction has processes in place to develop commercial agreements/financial Managed Entry Agreements (MEA) within their pricing and reimbursement/

\footnotetext{
${ }^{4}$ https://www.oegho.at/fileadmin/Benutzer/Aktuelles/qualitaetskrite rien-car-t-zentren-final_1.pdf.

5 Tisagenlecleucel (Kymriah) for Pediatric Acute Lymphoblastic Leukemia and Diffuse Large B-Cell Lymphoma I CADTH.ca.
} 


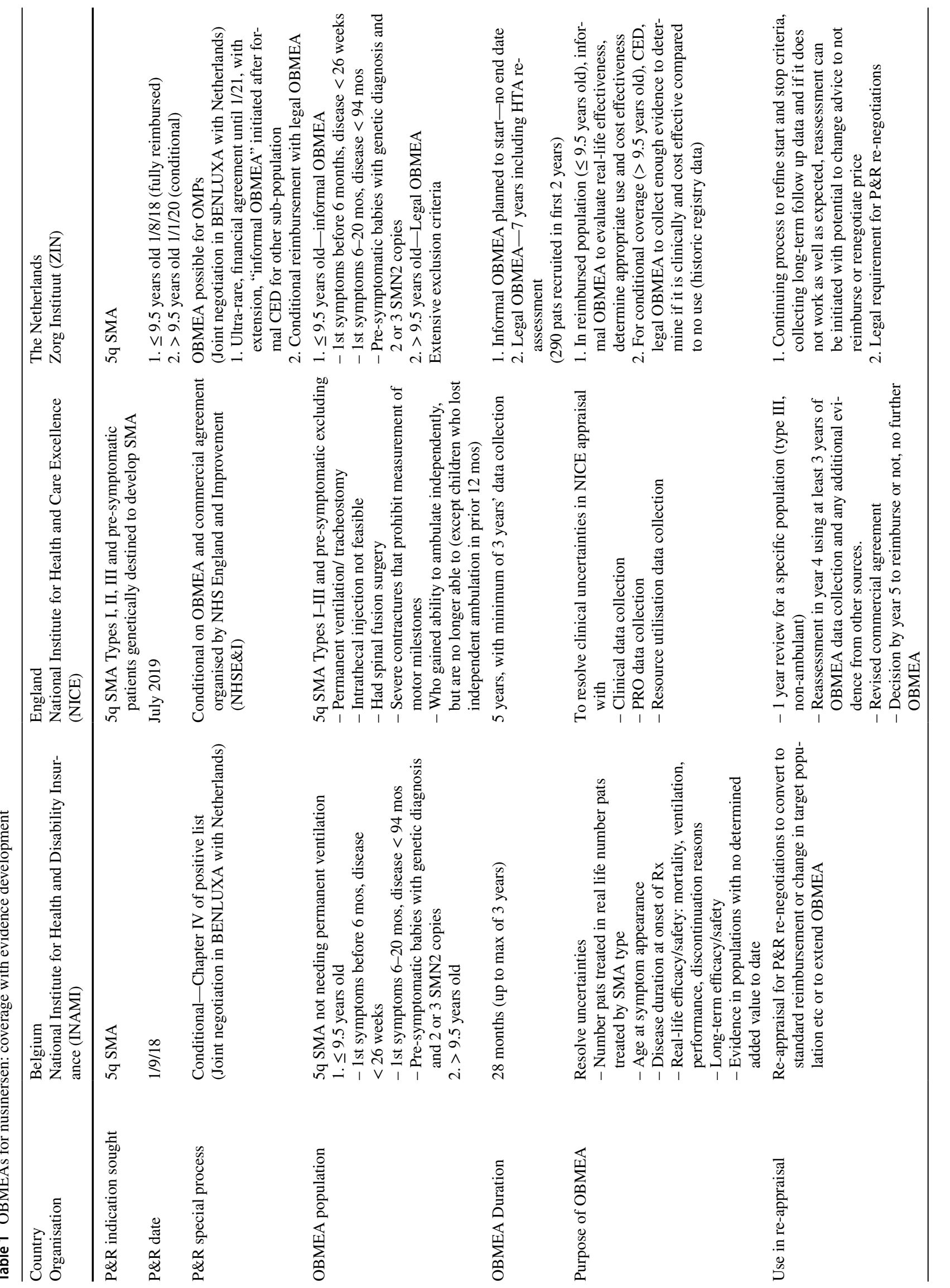




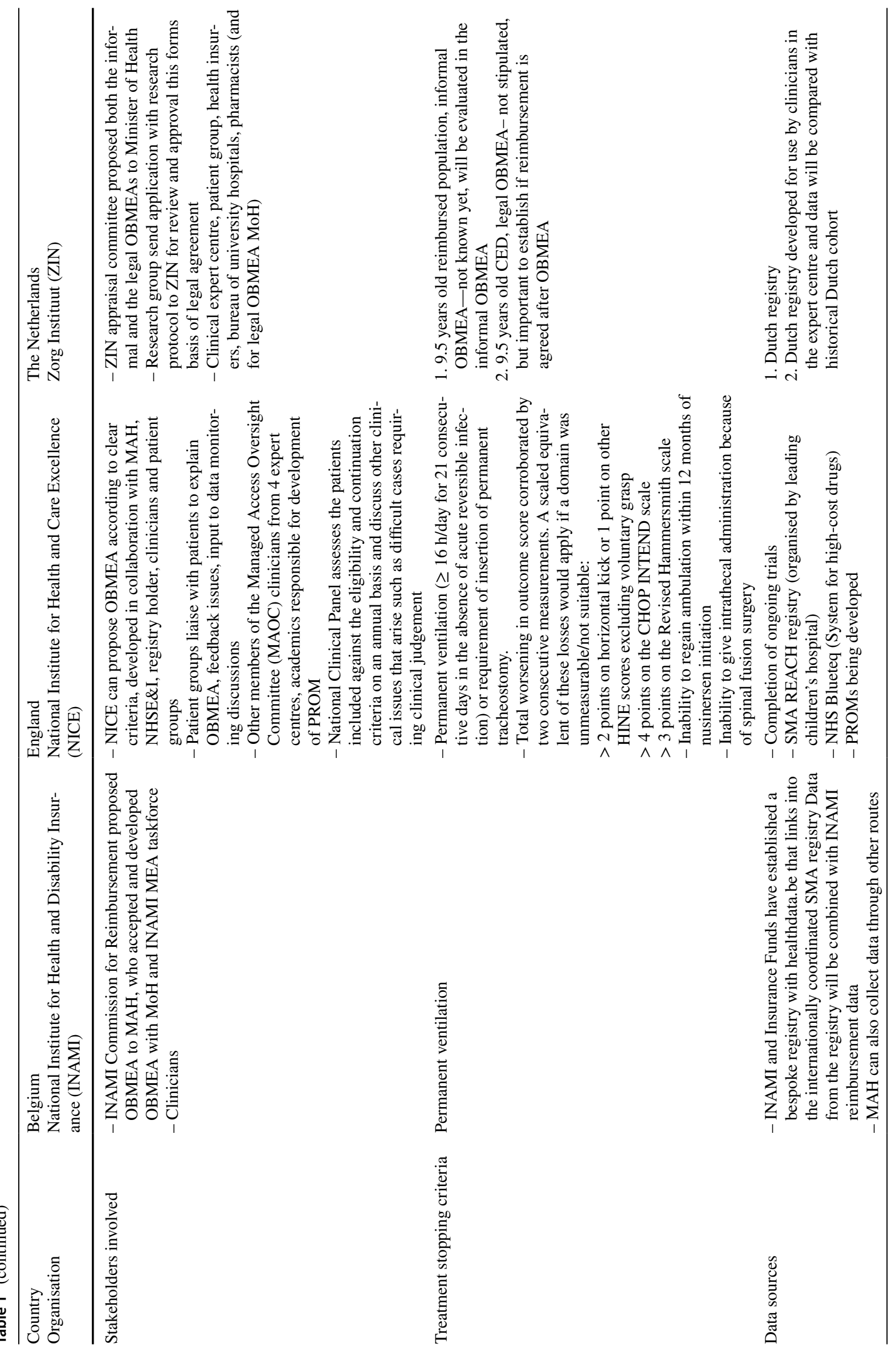




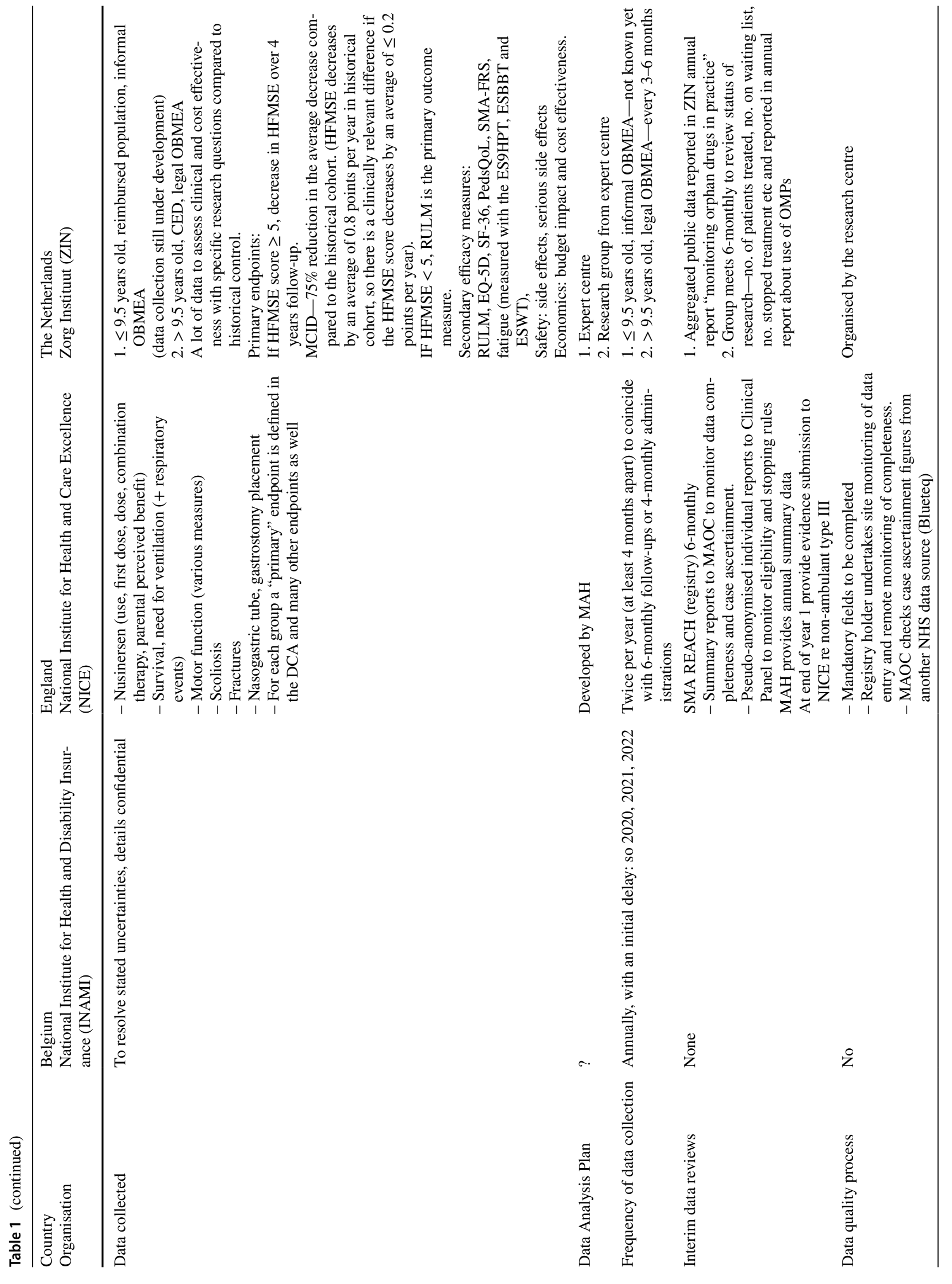




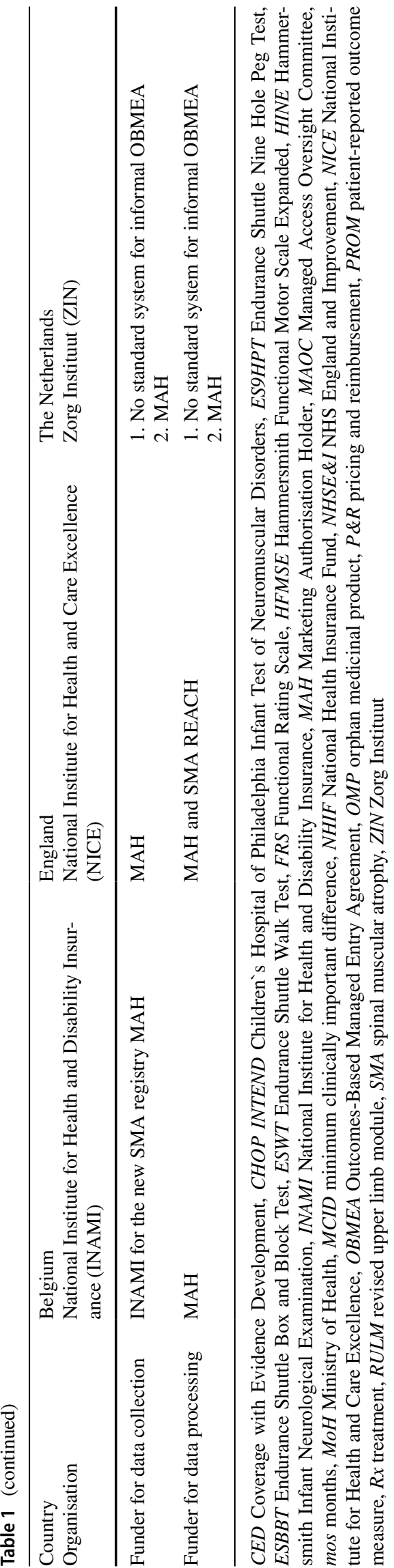

commissioning process, many of which have a clear legal underpinning. These commercial agreements are highly confidential and generally only involve the marketing authorisation holder and the payer/Ministry of Health and for our case studies, range from simple discounts to budget caps (Belgium, Bulgaria, Latvia [unvalidated], Lithuania, Spain).

Despite the confidentiality of these commercial agreements, public information was available for many jurisdictions to populate our OBMEA templates for the two case studies. As shown in Tables 1, 2, and 3, information was available about the rationale and design of the OBMEA in different countries covering topics that elucidate the purpose of the OBMEA, population given access, duration of agreement, stakeholder involvement, data to be collected and reporting mechanisms. These elements are presented in the following sections with augmentation of intelligence from the HTA expert workshops.

\subsubsection{Establishing the OBMEA}

Some countries have standing committees that oversee the establishment of all OBMEAs (such as Belgium) inviting in clinical/appraisal experts relevant to the treatment. In England, the case studies come from two separate programmes within NICE, the Cancer Drugs Fund and the HST programme. They had different processes for the establishment of OBMEAs, thus the form of data collection agreements is slightly different for the case studies (but rationalisation of these OBMEA processes is underway for the future).

Outcomes-based managed entry agreements are sometimes limited to specific groups of medicines such as (highcost) OMPs or those with conditional or exceptional marketing authorisations, nusinersen was considered via this route in The Netherlands. In some countries, OBMEAs are mandated for all medicines in a certain category, for example, in Italy those with a full innovation status must have a national registry that at least determines appropriate use (guiding eligibility for treatment and continuation criteria), as was the case for nusinersen, whereas tisagenlecleucel was the first product to undergo the "Payment at Results" OBMEAs in AIFA. In other countries, the use of an OBMEA for nusinersen and tisagenlecleucel was considered on a case-by-case basis to support appropriate use in individual patients and/or data collection to resolve clinical (and economic) uncertainties.

The OBMEAs for nusinersen were initiated between 2017 and 2020 and as shown by Whittal et al. [13] more evidence from studies in different sub-populations, clinical study follow-ups and real-world effectiveness might have been available for the later reimbursement decisions. This could have potentially resolved some uncertainties and impacted their need for OBMEA. For tisagenlecleucel, decisions were made at very similar times between January and August 2019. 


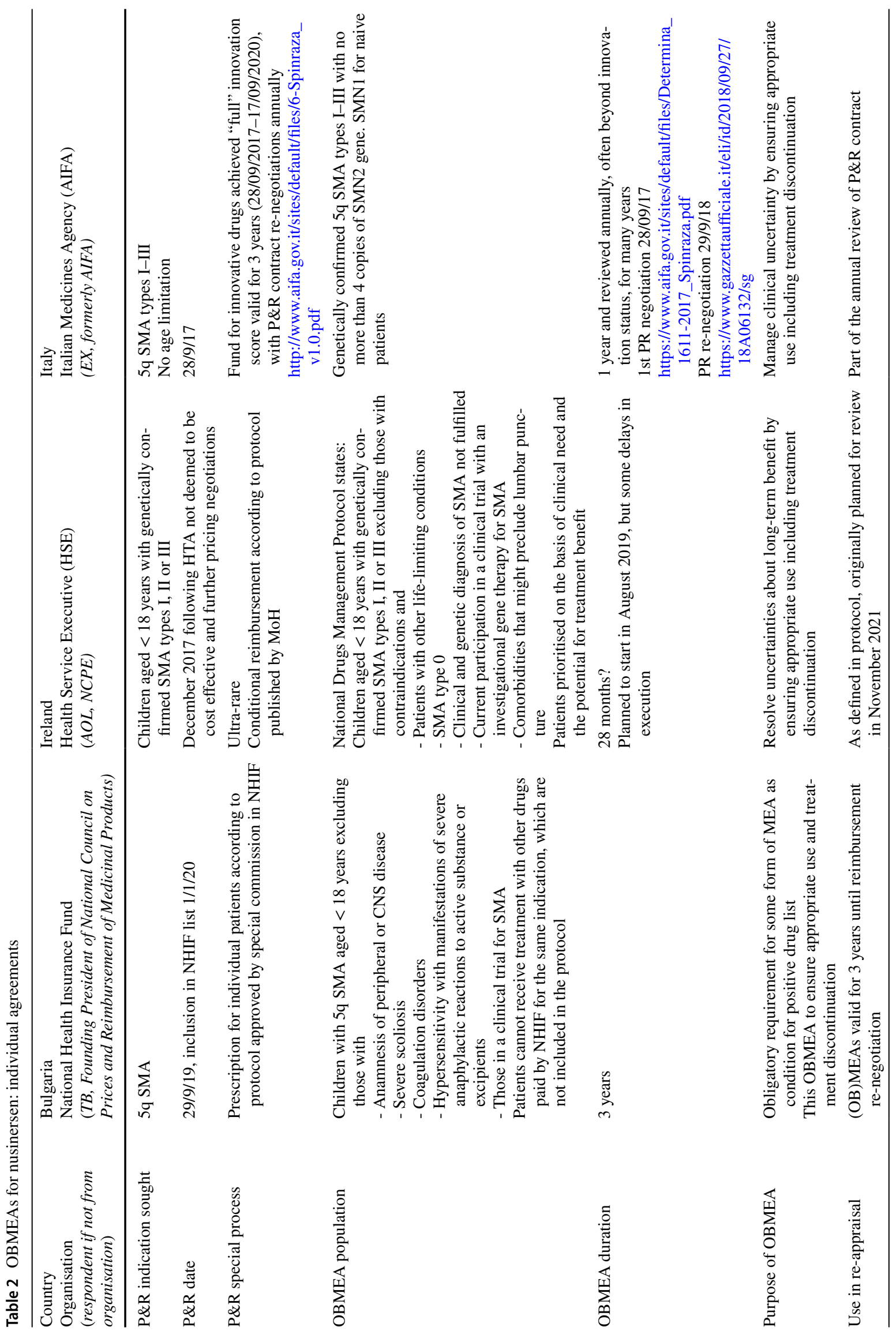




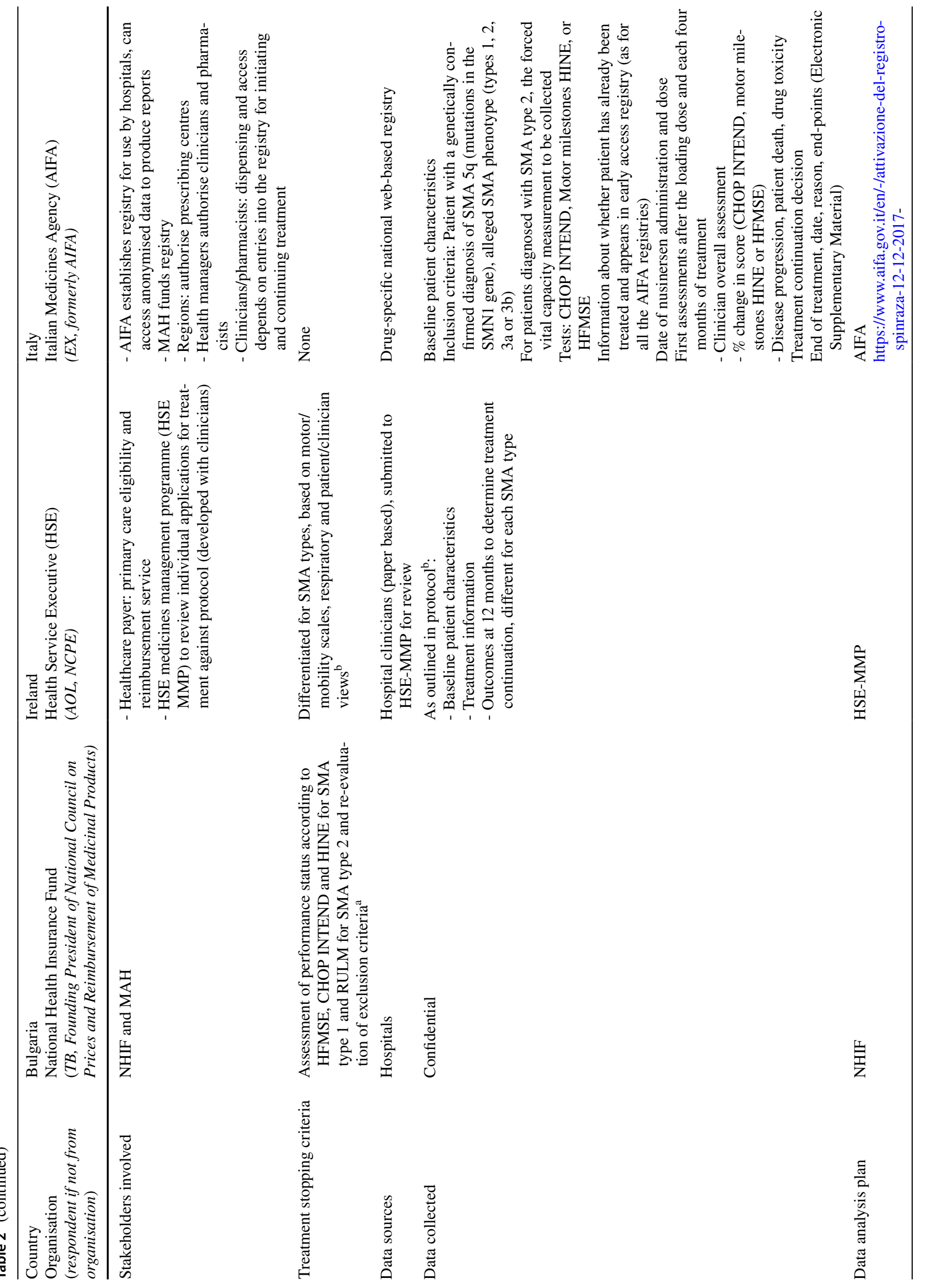




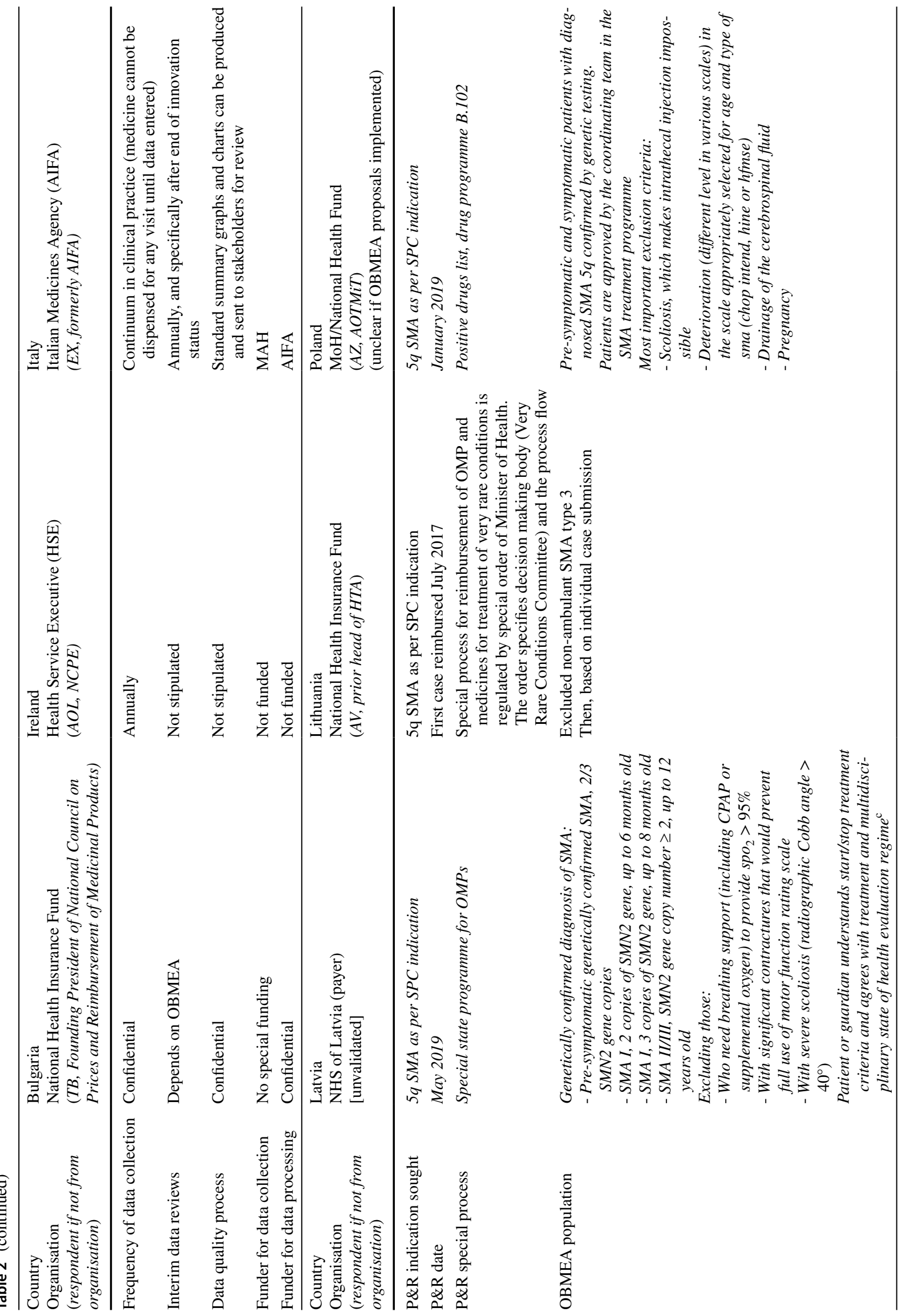




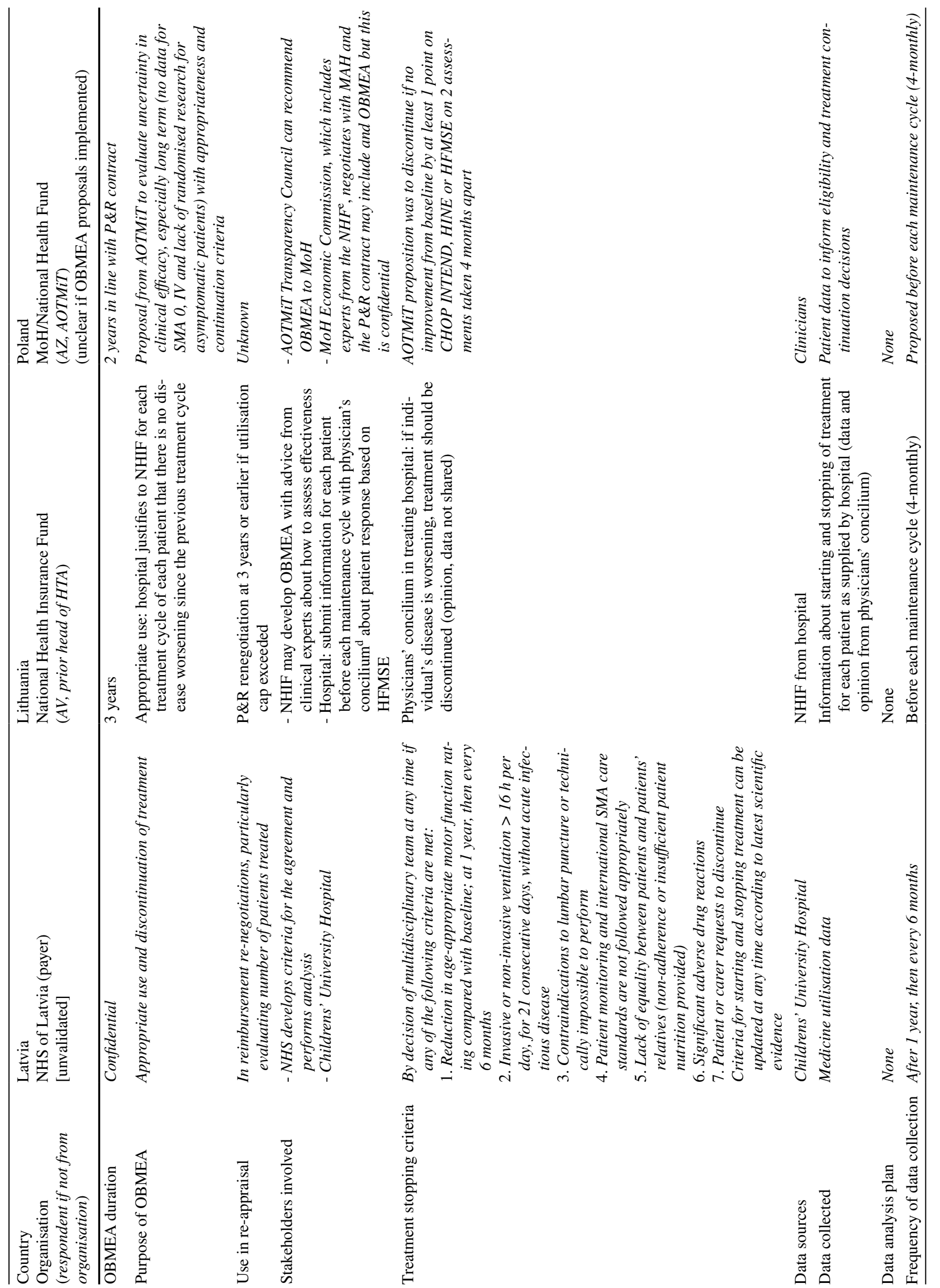




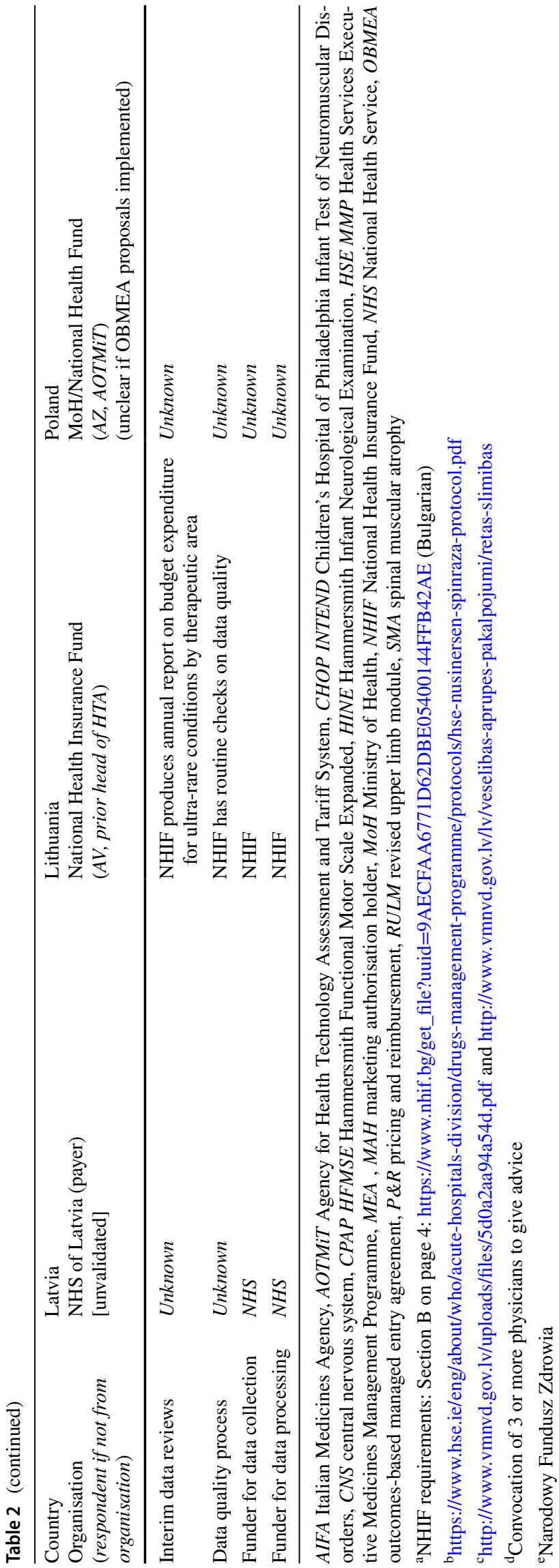

\subsubsection{Form of OBMEAs}

In most cases, the purposes of the OBMEAs for nusinersen and tisagenlecleucel were clear with specific clinical and/ economic uncertainties named relating to population characteristics, treatment administration, real-life and long-term efficacy, expensive concomitant or future treatments, and healthcare resource utilisation data. However, the link to appraisal reports, which would have been expected to be the documented source of the uncertainties, was not always clear.

For nusinersen, the individual-based OBMEAs in Table 2 show that they were generally used to collect outcomes to ensure "appropriate use" and determine treatment continuation. The table shows that even for these simpler forms of OBMEAs, a range of outcomes needs to be collected.

Only Italy and Spain implemented the more complex individual-based OBMEA that used individual outcomes to inform a staged payment model for tisagenlecleucel (Table 3). The population-based schemes in our case studies were illuminating because they included many elements of the individual-based schemes (with strict appropriateness criteria) but they went further to require data aggregation for re-analysis at a specific point in the future (Tables 1 and 3).

\subsubsection{OBMEA Population and Duration}

The reimbursed populations and inclusion criteria for the OBMEAs differ somewhat across countries. For nusinersen, Belgium simply refers to the regulatory authorised indication. Others state a variety of exclusion criteria that relate to ability to administer treatment (nusinersen), treatment stage and potential for response (tisagenlecleucel). These features are likely to feature in the clinical judgement of patient suitability in Belgium, even if not explicitly stated.

The differing age (and thus stage) restrictions for nusinersen probably arise from different value judgements made by appraisal committees about the extension of evidence from small trials in symptomatic young children to presymptomatic children and older patients. Two countries are of particular note.

As the indication for reimbursement sought to include all sub-types of SMA, this meant that in England, nusinersen was evaluated through the NICE Technology Appraisal programme. However, if a restriction had been made to type I, it would have probably been evaluated through the HST route (as was the case for onasemnogene abeparvovec). In the HST programme, a different decision-making criteria framework is used, including higher willingness-to-pay thresholds. In which case, an OBMEA may not have been needed, but access would have been restricted to specific patients.

As a pilot in the BENELUXA initiative, Belgium and the Netherlands published a joint assessment report on the 


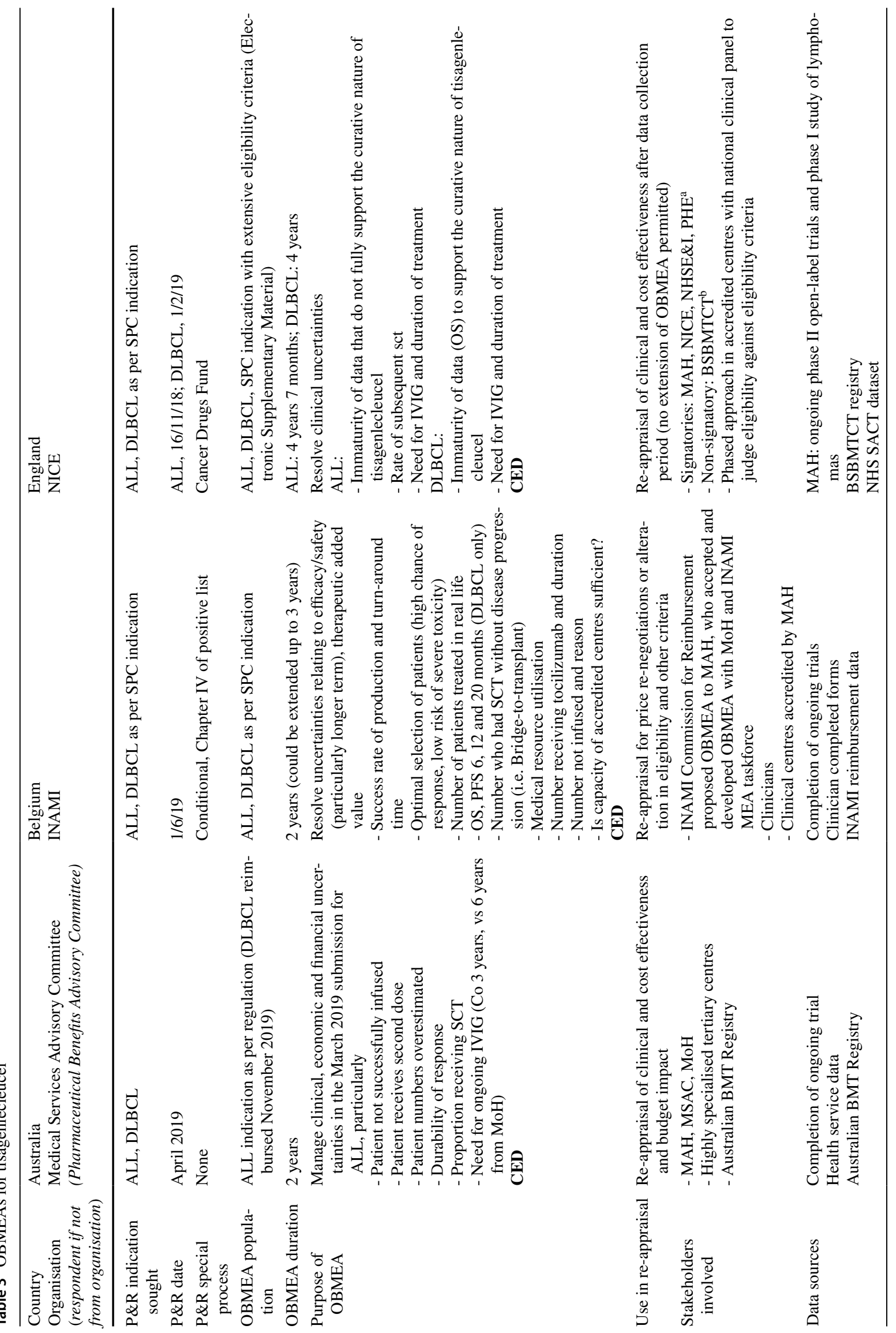




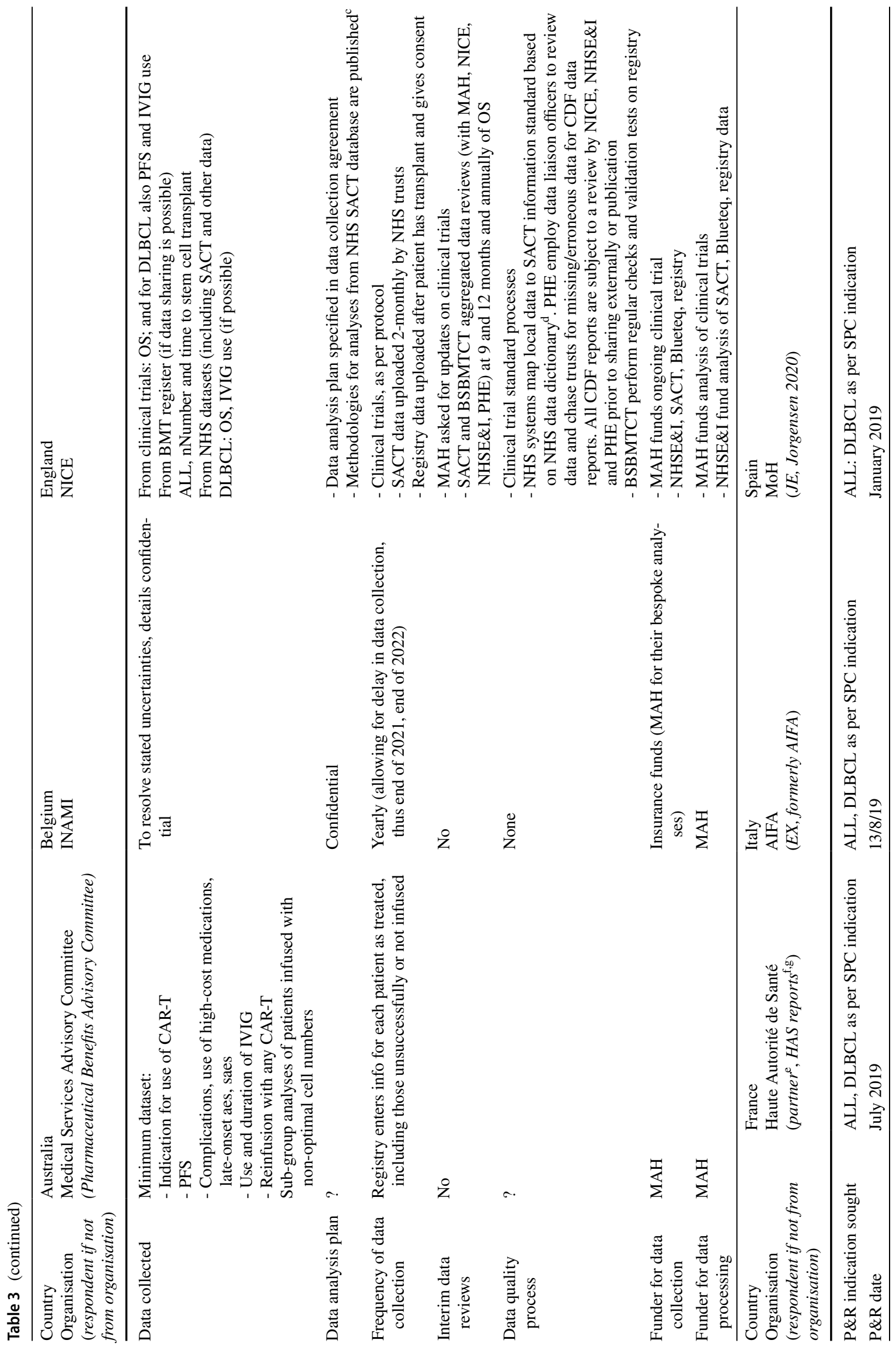




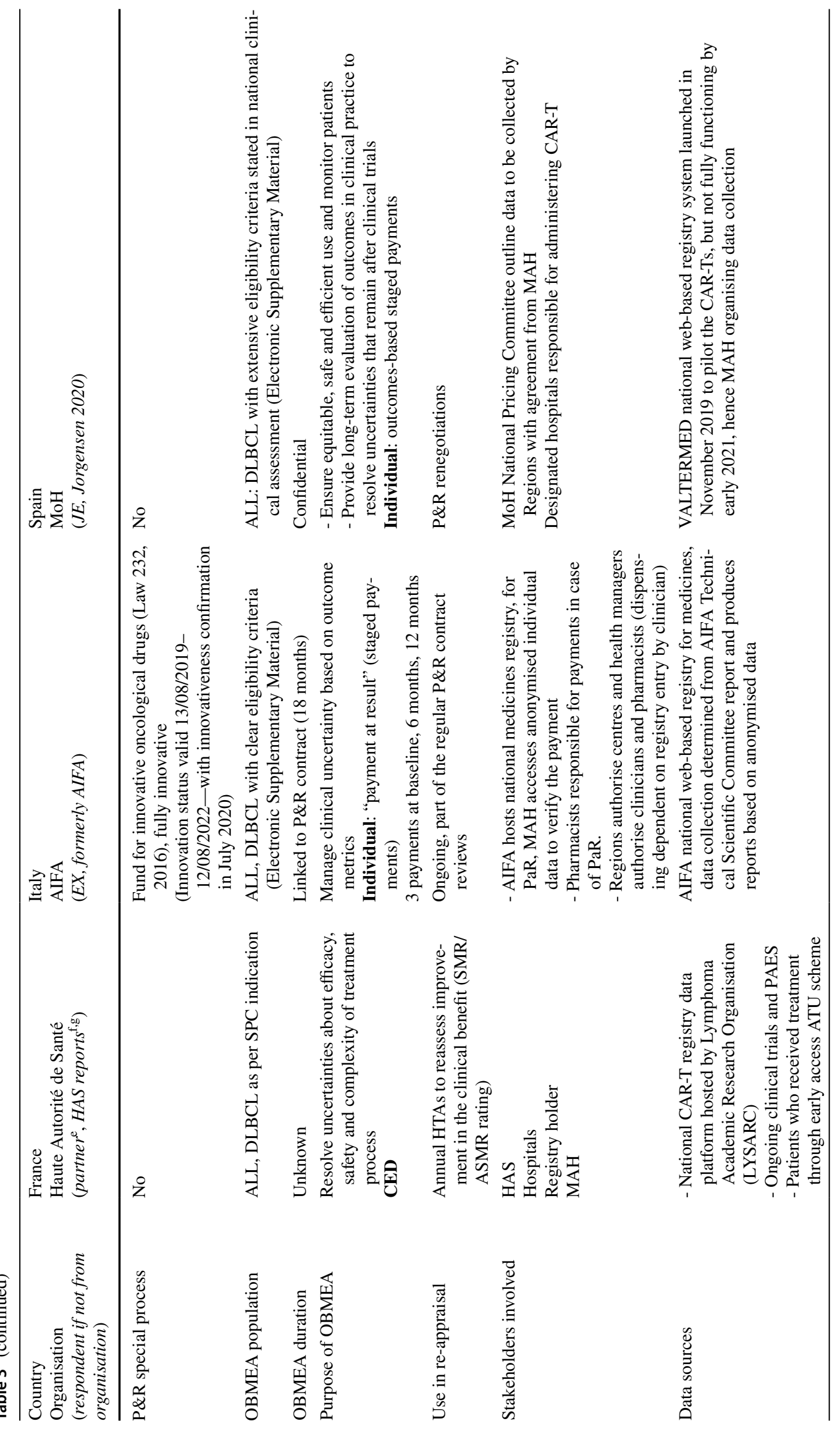




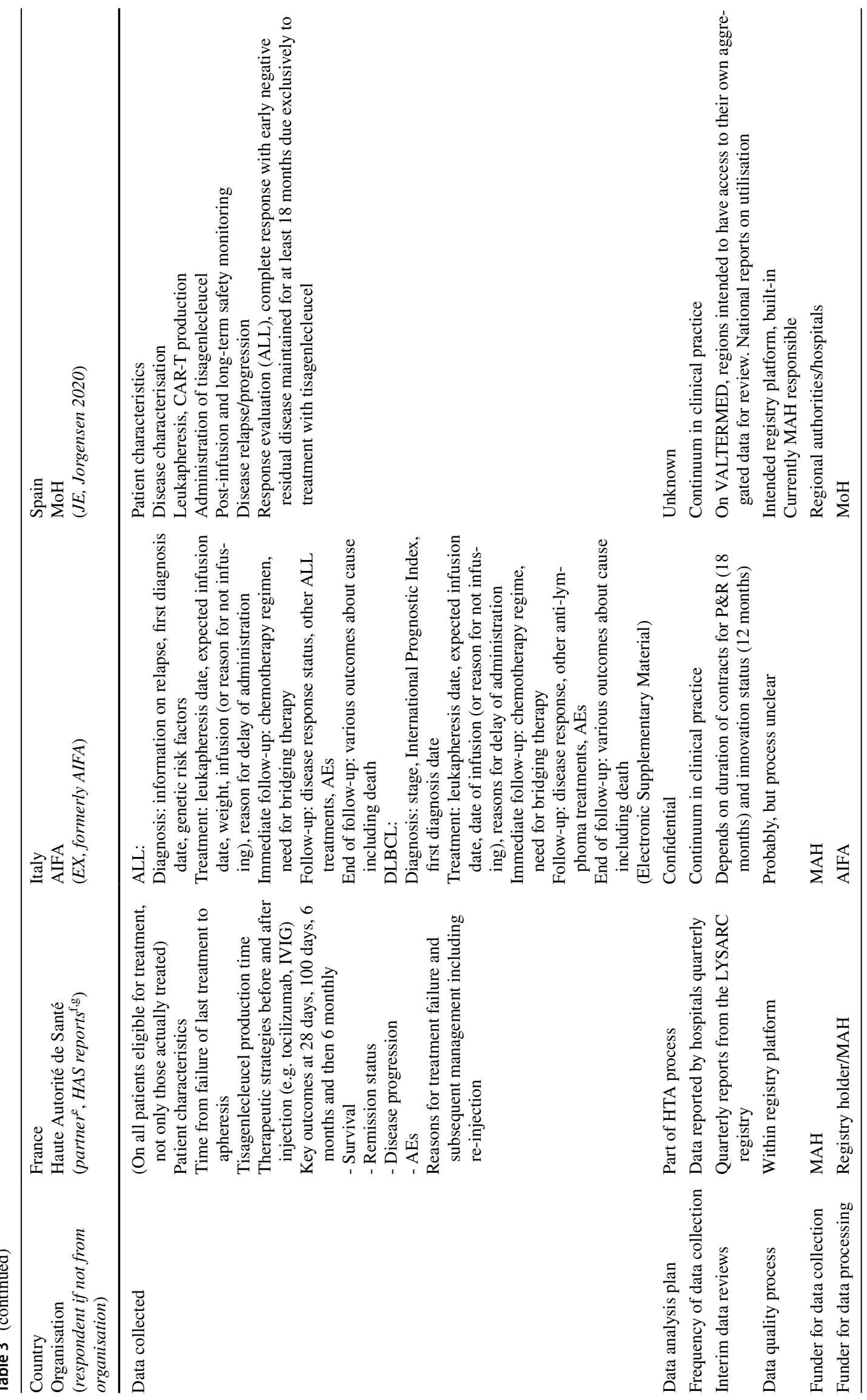




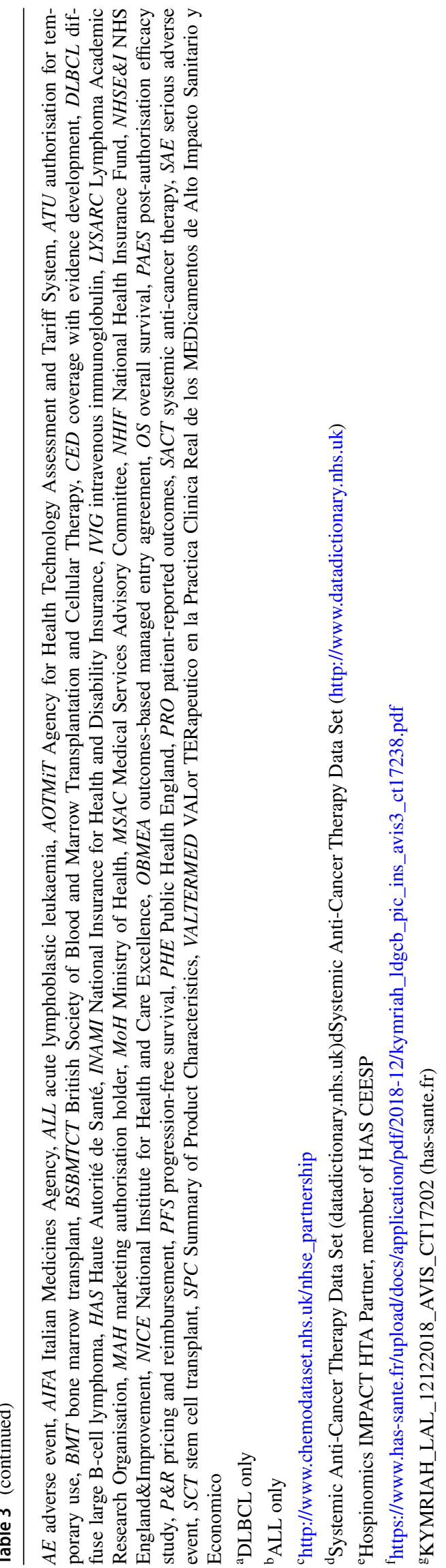

clinical and cost effectiveness of nusinersen. This informed a joint pricing negotiation, but each country implemented its own OBMEA as shown in Table 1. In the Netherlands, as the assessment report had identified that clinical research only included patients aged up to 9.5 years and that evidence was not transferable to older patients (SMA types II and III), different reimbursement decisions were made for subpopulations. For those aged 9.5 years and older, a legally based OBMEA is ongoing, whereby the health system will pay for nusinersen and its administration and the marketing authorisation holder must pay the costs of research to collect data to resolve stated uncertainties. For children aged under 9.5 years, reimbursement was agreed but, using a new arrangement for selected OMPs that seeks to evaluate real-life effectiveness and optimise treatment, an informal OBMEA (that does not involve the marketing authorisation holder) will start in 2021. In contrast, Belgium requested data collection on the entire population, but has no mandate to ensure these data are collected.

In all countries, treatment and data collection for both cases are assigned to a few expert centres that generally require some type of authorisation or accreditation. Only the Netherlands appears to have provided a sample size estimate for the legal OBMEA. In 2019, estimates were made of initially 170 and then 290 patients, with a recruitment period of 2 years. In February 2021, 300 patients had been screened and two-thirds of those were to be treated with nusinersen. For most other countries, the number of patients was explicitly named as a key uncertainty and other financial schemes were put in place to ensure budget caps.

Several countries have implemented OBMEAs with a duration of 2-3 years for these treatments, whereas in England, data collection is a minimum of 3 years to enable reappraisal within 4.5-5 years or earlier. In the Netherlands, a legal OBMEA with nusinersen is planned for 7 years, which goes beyond the usual 4-year period permitted, given the small number of patients anticipated.

\subsubsection{OBMEA Data Collection and Analysis}

Most countries are collecting data via a range of approaches that depend on each treatment, its uncertainties and the existing health data ecosystem infrastructure (clinical and/ or reimbursement or insurance data), with an assumption that ongoing clinical studies will be completed. For tisagenlecleucel, links have been made to existing chemotherapy datasets and national registries for bone marrow transplant, whereas for nusinersen links to clinical registries have been important or have needed to be established.

Italy and Spain have a national web-based platform for which bespoke data collection requirements are created for each medicine/therapeutic indication. This not only allows enforcement of eligibility and continuation criteria, but 
should encourage timely entry of data to access treatment. However, in Italy, the national platform is not linked to the hospital reimbursement system and thus duplicate data entry is required by the prescribers. In Spain, the platform is being established and was not fully functional for tisagenlecleucel.

Costs of OBMEA are unclear, apart from in the wellestablished system in Italy, where for an OMP registry the marketing authorisation holder pays approximately 30,000 Euros every three years, with a 10,000 Euros maintenance fee from the fourth year. This includes the construct of the data collection forms after the pricing and reimbursement negotiation, updates on data collection and aggregated standard reports.

Only England, the Netherlands and Spain stated an intention to capture patient-reported outcomes for these cases. In England, research was commissioned at the outset of the OBMEA for nusinersen to identify existing patientreported outcomes that could capture quality-of-life issues for patients with SMA. None adequately captured the issues that were considered important by patient representatives. As a result, the collection of patient-reported outcomes is yet to be operationalised. However, this data collection is a requirement of the English OBMEA and is expected to form part of the evidence submissions at the time of re-appraisal.

In England, the nusinersen OBMEA was undertaken within NICE's HST programme, which requires each patient/guardian and their treating physician to sign an informed assent form ${ }^{6}$. These not only provide consent for use of data, but also confirm understanding of the OBMEA process including the conditions under which treatment could be terminated and the implications of withdrawal (stopping treatment access).

Detailed data analysis plans were not in place in most countries for the nusinersen and tisagenlecleucel OBMEA at the outset. Interim data are understandably not intended to be published, but some intend to provide updates on progress in recruitment in annual reports about OMP use and most with CED intend to issue public reports after re-appraisal.

\section{Discussion}

Outcomes-based managed entry agreements arise from appraisal discussions or pricing and reimbursement negotiations to manage outstanding uncertainties (and high prices). The Organisation for Economic Co-operation and Development report [7] stated that little information is shared or published about OBMEAs in terms of treatments involved, OBMEA design and results (analyses and decision). This is

\footnotetext{
6 https://www.nice.org.uk/guidance/ta588/resources/managed-accessagreement-july-2019-pdf-6842812573.
}

important because OBMEAs require careful governance to address data ownership, audit, transparency and appeal [14].

Our research has found information about the purpose, duration and data to be collected in the OBMEAs for nusinersen and tisagenlecleucel in several jurisdictions. However, this information was only available following close interaction with experts (co-authors of this paper) who knew where to find this information on their national websites and who could help us translate key information. This has enabled a detailed comparison of the constructs of these OBMEA case studies, which provides valuable insights that can improve future use and implementation of OBMEAs with rare disease treatments.

Since this research was started, there has been increasing interest in approaches to manage uncertainties with the one-off cell and gene therapies, many of which have been developed for rare diseases. These therapies rely on benefits realisation in the future and thus innovative pricing models based on individual outcomes have been used in some cases [15-17]. Pricing models have not been the subject of this work as issues related to pricing are subject to confidentiality agreements. The goal of this work was to identify what OBMEA information was available for two specific cases and encourage further collaboration to support all jurisdictions and stakeholders in sharing what can easily be made public. Work around innovative pricing schemes will clearly continue and it will be important to consider it alongside this work on OBMEAs.

The nusinersen and tisagenlecleucel case studies demonstrate that OBMEAs are agreed, at a minimum, in negotiation with the marketing authorisation holder and the payer, and for CED in several countries, physicians and research groups are involved in the design of data collection. Only England and the Netherlands mentioned the involvement of patient groups. In the Netherlands, a covenant was signed by all stakeholders for the legal OBMEA (CED) of nusinersen. This gave written assurance to the Minister of Health that the OBMEA would be conducted so that it was capable of drawing conclusions about clinical effectiveness at the end of the agreement. This purposive approach to data collection showing the commitment of all stakeholders appears to be an important change in the Dutch process to ensure the effectiveness of the OBMEA and could be an important model for other systems.

Given the investment required from all stakeholders in the design and conduct of population-based schemes (CED), it is imperative that steps are taken to enable them to deliver meaningful results. They should have the rigour of a pragmatic trial with clear delineation of uncertainties to be resolved (research questions and associated outcomes), sample size determination (number of patients and duration of study), data management processes for data from all 
sources and statistical analysis plan. However, these aspects were not clear for all the CED examples in our case studies.

\subsection{Maximising Data Quantity and Quality}

The outcomes studied in the OBMEA case studies to inform eligibility, continuation and effectiveness decisions were based on measurements used in clinical trials, but not all were standard in clinical practice. This adds burdens for clinicians, patients and their families. However, in many countries, the OBMEA will result in more clinical data than was available in the original submission from the marketing authorisation holder. Hence, it is important to ensure the data, from both individual-based and population-based schemes are used not only to optimise care for the patient but also to contribute to health system efficiency by determining optimal treatment regimes.

Only The Netherlands stipulated a sample size for their OBMEA. This demonstrated how sample size considerations for an OBMEA go beyond that for a clinical study as the conditional reimbursement implies that all eligible patients should have access to treatment. In chronic rare diseases, the prevalent population will be eligible for study at the outset and those who should recieve treatment first in this cohort may need to be prioritised. When all have been offered treatment, only newly eligible cases will be available. When recruitment is complete for the OBMEA, consideration needs to be given as to what happens with other eligible patients. For rare diseases, it would seem valuable to study these patients and this was recognised in the Netherlands, where an additional study will be established after recruitment to the legal OBMEA (not funded by the marketing authorisation holder). In England, this is considered on a case-by-case basis and for nusinersen those not eligible for the OBMEA will form a comparative cohort with risk adjustment to allow comparison, if possible.

Although the data to be collected in the OBMEA were similar in different countries, the duration of agreements differed and the rationale for the duration was unclear. It may have been linked to decision-making milestones, such as pricing and reimbursement negotiations. Only the Netherlands clearly stated the expected recruitment period and duration of follow-up. Such information is important for CED and should be clearly documented, taking account of the prevalent and incident population in the health jurisdiction and the timing of the most important outcome assessments.

In summer 2020, EUnetHTA Joint Action 3 published its first Post-Launch Evidence Generation pilot data collection report for nusinersen [22]. Eight research questions addressed uncertainties in SMA sub-populations covering the number of patients treated, patient characteristics, treatment duration, dosing in different patient groups, quality-of-life and patient-reported outcomes, long-term safety and efficacy, and validation of new outcome measures for disease progression effects. A minimum dataset was then defined to collect relevant real-world data from nusinersen registries in the participating countries (Italy, Croatia, Finland, Norway, Portugal, The Netherlands). Outcomes were to be measured at baseline, 6 and 18 months and a brief statistical analysis plan was presented. As EUnetHTA Joint Action 3 comes to an end in 2021, it is unclear how well this study will progress and as can be seen from this case study research, countries have initiated their own data collection schemes for nusinersen.

Alongside bespoke data collection, marketing authorisation holders were expected to continue their planned clinical programmes for the case studies, completing ongoing studies, and providing longer term follow-up and information about various sub-populations. However, none of the OBMEAs referred to regulatory post-licensing data requirements and how that may bolster evidence in the future and be used alongside data arising from the OBMEA.

To maximise information coming from disparate sources, linking of records is needed. This can be more easily achieved within health systems for secondary use of administrative data, where consent is presumed in law. However, challenges can arise when linkage to other sources, such as clinical registries is required. The AIFA registry is a standalone system that does not link to other data, which is seen as a limitation by many countries that have good electronic health records. For this reason, the new VALTERMED medicines' registry system in Spain is of particular interest, not only because of its national status, but because it intends to include patient-reported outcomes and to link to other health service data.

Several countries are using national registries to collect data, SMA disease registries for nusinersen and stem cell transplantation registries for tisagenlecleucel. The aim is to link to individual patient data and thus national registries are preferred. However, as the RWE4Decisions initiative states [9], more needs to be done to bring the HTA/payer and clinical communities together to ensure that clinical registries are aligned with HTA/payer needs. This needs to include consideration of how the European registries for rare diseases being developed by the European Reference Networks can be used in jurisdictions.

Our case studies also show that there is opportunity for HTA/payers to collaborate across jurisdictions to not only align their appraisals to come to a common agreement on decision-relevant uncertainties, but to also agree a core dataset for data collection post-HTA. This could substantially improve timeliness and cost and efficiency of data collection [3] and optimise re-appraisal. However, it is difficult to achieve in practice. The BENELUXA joint assessment of nusinersen led to agreement on the need for an international 
SMA registry, but despite this, each country of that partnership developed its own OBMEA as outlined in Tables 1 and 2. The challenges were also recognised by EUnetHTA who stated that for rare diseases "collaboration among Member States in the definition of the minimum dataset and in gathering evidence coming from different registries and databases is crucial for HTA" [18].

In the national web-based OBMEA systems, data checking elements are embedded and automatic summary reports are produced that update on the level of data collection. Where a variety of data sources is used and several treatment centres are involved, careful monitoring processes are needed if sufficient data are to be collected, but not all countries have organised this for the case studies. This is essential not only to ensure data quality but to monitor patient recruitment and data completeness of all required assessments. NICE uses a multi-stakeholder Managed Access Oversight Committee for nusinersen. This committee meets every 6 months to discuss data collection issues that arise in clinical practice and monitors completeness and quality of data. One challenging issue that had to be faced in 2020 was the high level of missing data as a result of cancelled clinic visits because of the COVID-19 pandemic. As a result, alternative treatment continuation protocols have been developed and data analysis plans modified.

\subsection{Capturing Patients' Perspectives}

In the field of rare diseases, knowledge about the disease evolves, particularly when the first disease-modifying medicine comes to market in an area of high unmet need. Therefore, evidence generation post-reimbursement should not only seek to resolve uncertainties found in the HTA, but also to capture new knowledge about the disease (particularly important if comparisons are made to natural history) and optimisation of treatment in real life. For this, involvement of patients and their informal caregivers would seem essential. However, there was little evidence of patient involvement in the OBMEA case studies. A few of the OBMEAs indicated the plan to collect patient-reported outcomes, which are considered particularly important in rare diseases $[19,20]$. However, there is often no suitable patient-reported outcome for a rare disease (as was the case for nusinersen in England) and thus other mechanisms are needed to capture patients' perspectives. As part of this project, KF/EK/SU have worked with the patient group leaders involved in NICE OBMEAs (including for spinal muscular atrophy) to develop a patient group submission template for re-appraisal. This will enable patient groups to gather evidence from patients about the conduct of the OBMEA and the benefits and disbenefits of treatment that are not captured in the quantitative data collection [21].

\subsection{Examples of Successful OBMEA}

Coverage with evidence development schemes have been criticised for their inability to collect sufficient good quality data in a timely manner to inform re-appraisal/pricing and reimbursement re-negotiations. As our case studies were about the establishment and implementation of OBMEAs, not re-appraisal, examples of OBMEAs that had made a difference were discussed in the expert workshops. Australian experts shared examples of OBMEAs for four rare cancers and one for lumacaftor/ivacaftor in cystic fibrosis. Of these, two are ongoing, one was suspended by the sponsor, one led to a change in reimbursement population and one led to no change.

In the English Cancer Drugs Fund, brentuximab (NICE TA524) and obinutuzumab (NICE TA629) for the treatment of rare cancers have completed their OBMEAs and resulted in full reimbursement. Elosulfase alfa for mucopolysaccharidosis type IVA was the first OBMEA enacted in the NICE HST programme. The treatment was due to be re-appraised in December 2020, but this has been delayed. The scope of the re-appraisal was agreed with stakeholders in December 2019. However, in February 2020, the re-appraisal was suspended "because the company has not provided an evidence submission that is adequate for the committee to make a decision and will not accept the terms of the charging procedure for HSTs" ". One challenge with this re-appraisal is that it will now be undertaken under a different decision-making process. Elosulfase alfa (and the other early appraisals) did not consider cost effectiveness and willingness-to-pay thresholds, but these are now clearly stated in their methods ${ }^{8}$.

Thus, the jury is still out as to whether OBMEA can be successful for rare disease treatments, but perhaps through greater collaboration among HTA/payers and with stakeholders, there can be more transparency about uncertainties that exist, constructs for data collection, and sharing of results that will optimise treatment and improve health service efficiency.

In Italy, where the majority of OBMEAs are individualbased schemes, changes have related to confidential price re-negotiations or optimisation of use, such as alteration of continuation criteria. Only a few treatments have been withdrawn from reimbursement and this was as a result of safety issues raised by regulators ${ }^{9}$ [11]. Given the increasing complexity of treatments coming to market, authors agree

\footnotetext{
${ }^{7}$ Project information I Elosulfase alfa for treating mucopolysaccharidosis type IVa (review of HST2) [ID1643] I Guidance I NICE.

8 https://www.nice.org.uk/Media/Default/About/what-we-do/NICEguidance/NICE-highly-specialised-technologies-guidance/HST-inter im-methods-process-guide-may-17.pdf.

${ }^{9}$ Lartruvo (olaratumab): https://www.aifa.gov.it/en/-/chiusura-regis tro-lartruvo-nota-informativa-importante.
} 
there is value in the use of OBMEAs for optimisation of use, to perhaps alter the eligible population or criteria for treatment continuation, and to ensure good organisation of care such as specialist centre capacity and to monitor the quality of care in each centre.

\subsection{New National OBMEA Initiatives}

In 2019, Germany passed the GSAV law that that gives the Federal Joint Committee increased authority to impose data collection requirements and price reductions if data do not support added value. It is anticipated this will be used for OMPs, but no further guidance has been issued. In 2019, Scotland also implemented a new "ultra-orphan pathway" enabling designated medicines to have an initial HTA review by the Scottish Medicines Consortium to identify key uncertainties, then requiring the marketing authorisation holder to submit a 3-year data collection plan to the Scottish Government to resolve the uncertainties. Given the small size of Scotland, it is likely that the subsequent re-appraisal will include data beyond Scotland, but this process is not transparent and few details are available.

\section{Conclusions}

These case studies show that OBMEAs are being implemented to manage uncertainties relating to the real-life and long-term clinical effectiveness and inform economic evaluations of rare disease treatments in several jurisdictions. This research focuses on understanding how the challenges in the establishment and implementation of OBMEAs for two different forms of rare disease treatment (nusinersen and tisagenlecleucel) have been addressed to deliver data of sufficient quantity and quality to inform re-appraisal.

During the IMPACT HTA WP10 research programme, a strong message has emerged from all stakeholders that OBMEAs should not become routine practice, as their implementation is burdensome for all parties. This aligns with past research that has queried the sustainability and effectiveness of OBMEAs [4] and suggested that OBMEAs should be the exception and not the norm. This was strongly reiterated by all involved in this case study research.

If OBMEAs are to be a credible conditional reimbursement route for rare disease treatments, the costs and feasibility of collecting sufficient data to inform decisions must be scrutinised at the outset, then steps must be undertaken to ensure data quality and completeness. One important mechanism to achieve this could be a covenant agreed by all stakeholders providing assurance that all necessary actions will be taken to ensure that the data collected will be sufficient for decision making as implemented in The Netherlands for nusinersen.
Bringing together the learnings about establishment of OBMEAs for these two case studies of rare disease treatments with previous literature about when to conduct an OBMEA, the IMPACT HTA WP10 research team has developed a checklist to determine the feasibility of undertaking an OBMEA for a rare disease treatment [23]. This checklist could be used by an individual jurisdiction, or across jurisdictions, as for some rare diseases (particularly the ultrarare), collaborative approaches seem essential. Collaboration could foster discussions at the time of appraisal to determine decision-relevant uncertainties and a minimum dataset to be collected by all and shared in aggregated form for future reappraisal. IMPACT HTA WP10 has issued tools to support implementation of OBMEA [24, 25] that could help standardise national approaches and support collaboration, but the tools need to be piloted, and adapted with experience.

Another important element to ensuring the efficiency and effectiveness of OBMEAs for rare disease treatments is to encourage transparency in reporting the plans for, interim updates on status and results of OBMEAs. Such information should be publicly available and thus perhaps, the HTA database, now hosted by the International Network of Agencies for HTA, could be enhanced to host such information. As the Organisation for Economic Co-operation and Development indicates [7], such sharing could reduce duplication of effort across countries and enable learning, which is particularly important for rare disease treatments.

Supplementary Information The online version contains supplementary material available at https://doi.org/10.1007/s40273-021-01050-5.

Acknowledgements We are grateful to Mike Drummond and Carlo Federici, Bocconi University, Italy for sharing emerging work from the COMED project about Coverage with Evidence Development processes. We thank Karen Binnecamp, and Andrew Mitchell, Australian Government Department of Health (supporting the Australian Pharmaceutical Benefits Advisory Committee), for contributing reports that allowed the summary table to be completed and for contributing to one workshop. We thank the following for completing templates: Elka Boncheva, Sofia University, Bulgaria; Jean-Claude K. Dupont, Hospinnomics, France; Zinta Rugaja, NHS, Latvia; Claudia Wild, Austrian Institute for HTA, Austria.

\section{Declarations}

Funding Jaime Espin, Karen M. Facey, Elena Nicod, Entela Xoxi, Sheela Upadhyaya and Anna Zaremba were funded for this research from the European Commission's Horizon 2020 research and innovation programme for the IMPACT-HTA project (grant number 779312). The results presented here reflect the authors' views and not the views of the European Commission. The European Commission is not liable for any use of the information communicated. The other authors did not receive funding for this research.

Conflicts of Interest Karen M. Facey, Jaime Espin and Entela Xoxi have received fees from various pharmaceutical companies for speaking and advisory roles, none of which relates to the topics in this pa- 
per. Elena Nicod is employed part-time by Dolon Ltd, no conflicts arise with this work. Emma Kent, Angèl Link, Elena Nicod Aisling O'Leary, Inneke van de Vijver, Anna Zaremba, Tatyana Benisheva, Andrius Vagoras and Sheela Upadhyaya have no conflicts of interest that are directly relevant to the content of this article.

Ethics Approval No ethical approval was required for this study.

Consent to Participate Completed templates included consent.

Consent for Publication All authors have provided consent for publication.

Availability of Data and Material All data are provided in Tables 1,2 and 3, no programming has been undertaken.

Code Availability Not applicable.

Authors' Contributions EX, EN, SU and KF developed the case study template. EN issued invitations to complete the template. KF/SU contacted marketing authorisation holders to determine which countries might have OBMEAs. All authors completed a template and answered queries from KF. KF amalgamated responses and all authors (except $\mathrm{TB}$ and $\mathrm{AV}$ ) contributed to several discussion meetings about results, clarification of processes and key messages. KF led the writing of the paper and all authors critically reviewed drafts and approved the manuscript.

Open Access This article is licensed under a Creative Commons Attribution-NonCommercial 4.0 International License, which permits any non-commercial use, sharing, adaptation, distribution and reproduction in any medium or format, as long as you give appropriate credit to the original author(s) and the source, provide a link to the Creative Commons licence, and indicate if changes were made. The images or other third party material in this article are included in the article's Creative Commons licence, unless indicated otherwise in a credit line to the material. If material is not included in the article's Creative Commons licence and your intended use is not permitted by statutory regulation or exceeds the permitted use, you will need to obtain permission directly from the copyright holder. To view a copy of this licence, visit http://creativecommons.org/licenses/by-nc/4.0/.

\section{References}

1. Nicod E, Annemans L, Bucsics A, Lee A, Upadhyaya S, Facey K. HTA programme response to the challenges of dealing with orphan medicinal products: process evaluation in selected European countries. Health Policy. 2019;123:140-51.

2. Nicod E, Whittal A, Drummond M, Facey K. Are supplemental appraisal/reimbursement processes needed for rare disease treatments? An international comparison of country approaches. Orphanet J Rare Dis. 2020;15:1-14.

3. Bouvy JC, Sapede C, Garner S. Managed entry agreements for pharmaceuticals in the context of adaptive pathways in Europe. Front Pharmacol. 2018;9:280.

4. Klemp M, Frønsdal KB, Facey K. What principles should govern the use of managed entry agreements? Int J Technol Assess Health Care. 2011;27:77-83.

5. Boggild M, Palace J, Barton P, Ben-Shlomo Y, Bregenzer T, Dobson C, et al. Multiple sclerosis risk sharing scheme: two year results of clinical cohort study with historical comparator. BMJ. 2009;339:1359-63.
6. Carlson JJ, Chen S, Garrison LP. Performance-based risk-sharing arrangements: an updated international review. Pharmacoeconomics. 2017;35:1063-72.

7. Wenzl M, Chapman S. Performance-based managed entry agreements for new medicines in OECD countries and EU member states: how they work and possible improvements going forward. OECD Health Work Pap. 2019. https://doi.org/10.1787/6e5e4 c0f-en.

8. Morel T, Arickx F, Befrits G, Siviero P, Van Der Meijden C, Xoxi $\mathrm{E}$, et al. Reconciling uncertainty of costs and outcomes with the need for access to orphan medicinal products: a comparative study of managed entry agreements across seven European countries. Orphanet J Rare Dis. 2013;8:198.

9. Facey KM, Rannanheimo P, Batchelor L, Borchardt M, De Cock J. Real-world evidence to support payer/HTA decisions about highly innovative technologies in the EU: actions for stakeholders. Int $\mathrm{J}$ Technol Assess Health Care. 2020;3:1-10.

10. Reckers-Droog V, Federici C, Brouwer W, Drummond M. Challenges with coverage with evidence development schemes for medical devices: a systematic review. Heal Policy Technol. 2020;9:146-56. https://doi.org/10.1016/j.hlpt.2020.02.006.

11. Xoxi E, Facey K, Americo C. The evolution of AIFA registries to support managed entry agreeements for orphan medicinal products in Italy. Front Pharmacol. 2021 (Accepted June 2021)

12. Jørgensen J, Hanna E, Kefalas P. Outcomes-based reimbursement for gene therapies in practice: the experience of recently launched CAR-T cell therapies in major European countries. J Mark Access Health Policy. 2020;8:1715536. https://doi.org/10.1080/20016 689.2020 .1715536$.

13. Whittal A, Nicod E, Drummond M, Facey K. Examining the impact of different country processes for appraising rare disease treatments: a case study analysis. Int J Technol Assess Health Care. 2021;37:e65. https://doi.org/10.1017/S0266462321000337.

14. MacLeod S, Mitton C. Editorial: we know accurately only when we know little. Pharmacoeconomics. 2010;28:105-7.

15. Coyle D, Durand-Zaleski I, Farrington J, Garrison L, von der Schulenburg JMG, Greiner W, et al. HTA methodology and value frameworks for evaluation and policy making for cell and gene therapies. Eur J Health Econ. 2020;21:1421-37. https://doi.org/ 10.1007/s10198-020-01212-w.

16. Fiorenza S, Ritchie DS, Ramsey SD, Turtle CJ, Roth JA. Value and affordability of CAR T-cell therapy in the United States. Bone Marrow Transplant. 2020;55:1706-15. https://doi.org/10.1038/ s41409-020-0956-8.

17. Massachusetts Institute of Technology. Precision financing solutions for durable/potentially curative therapies. Massachusetts Institute of Technology: Center for Biomedical Innovation [Internet]. 2019 Available from MIT FoCUS Precision Financing 2019F 201v023.pdf. Accessed 11 June 2021.

18. EUnetHTA Joint Action 3. EUnetHTA WP5B PLEG pilot on nusinersen (Spinraza): common evidence gaps report. 2020. https://eunethta.eu/wp-content/uploads/2020/05/EUnetHTAPLEG_FP_01_Nusinersen_Common-Evidence-Gaps-report.pdf. Accessed 26 May 2021.

19. Whittal A, Meregaglia M, Nicod E. The use of patient-reported outcome measures in rare diseases and implications for health technology assessment. Patient. 2021. https://doi.org/10.1007/ s40271-020-00493-w.

20. Meregaglia M, Whittal A, Nicod E, Drummond M. 'Mapping' health state utility values from non-preference-based measures: a systematic literature review in rare diseases. Pharmacoeconomics. 2020;38:557-74.

21. IMPACT_HTA_WP10. Patient group submissions template for re-appraisal after OBMEA. 2021. https://8c3e11d9-5f36-452fabe3-c95befd6e85d.filesusr.com/ugd/e1a359_9937a09bdf2141d 
6aaca097b488c233f.docx?dn=21033IMPACT_HTA_WP10O BMEAPatientGr. Accessed 26 May 2021.

22. EUnetHTA Joint Action 3. EUnetHTA WP5B PLEG pilot on nusinersen $\left(\right.$ Spinraza $\left.^{\circledR}\right)$ minimum data set report. 2020. https:// eunethta.eu/wp-content/uploads/2020/07/EUnetHTA-PLEG_FP_ 01_Nusinersen_Minimum-data-set_report.pdf. Accessed 26 May 2021.

23. IMPACT_HTA_WP10. Checklist for a rare disease treatment is an outcomes-based managed entry agreement feasible? 2021. https://8c3e11d9-5f36-452f-abe3-c95befd6e85d.filesusr.com/ugd/ e1a359_b215b0e1c94243668237a883a0c66395.pdf. Accessed 26 May 2021.
24. IMPACT_HTA_WP10. IMPACT HTA WP10 OBMEA template. 2021. https://8c3e11d9-5f36-452f-abe3-c95befd6e85d.filesusr. com/ugd/e1a359_c2b953dd235348c7802785d65a745e9f.docx? $\mathrm{dn}=210331 \% 20 \mathrm{IMPACT}$ _HTA_WP10\%20OBMEA\%20Tem plate.do. Accessed 26 May 2021.

25. IMPACT_HTA_WP10. Terms of reference for a monitoring committee responsible for overseeing implementation of an OBMEA. 2021. https://8c3e11d9-5f36-452f-abe3-c95befd6e85d.filesusr. com/ugd/e1a359_729ae466e9bb47cc8c2db5f62f4efcf1.docx?dn= 210331IMPACT_HTA_WP10OBMEAMonitoring. Accessed 26 May 2021.

\section{Authors and Affiliations}

\section{Karen M. Facey ${ }^{1}$ (D) Jaime Espin ${ }^{2,3,4}$ (D) Emma Kent ${ }^{5}$ (D) $\cdot$ Angèl Link $^{6} \cdot$ Elena Nicod $^{7}$ (D) Aisling O'Leary $^{8}$. Entela Xoxi ${ }^{9}$ - Inneke van de Vijver ${ }^{10} \cdot$ Anna Zaremba $^{11} \cdot$ Tatyana Benisheva $^{12}$ (D) Andrius Vagoras $^{13}$ (D) Sheela Upadhyaya ${ }^{5}$}

Karen M. Facey

karen.facey@ed.ac.uk

1 Usher Institute, University of Edinburgh, NINE Edinburgh Bioquarter, 9 Little France Road, Edinburgh 16 4UX, EH, UK

2 Andalusian School of Public Health/Escuela Andaluza de Salud Pública (EASP), Granada, Spain

3 CIBER of Epidemiology and Public Health (CIBERESP), Madrid, Spain

4 Instituto de Investigación Biosanitaria ibs, Granada, Spain

5 National Institute for Health and Care Excellence (NICE), London, UK

6 Zorginstituut (ZIN) Nederland, Diemen, The Netherlands
7 Centre for Research on Health and Social Care Management (CERGAS), Bocconi University, Milan, Italy

8 National Centre for Pharmacoeconomics (NCPE), Trinity Centre for Health Sciences, St. James's Hospital, Dublin, Ireland

9 Università Cattolica del Sacro Cuore, Rome, Italy

10 National Institute for Health and Disability Insurance (INAMI), Brussels, Belgium

11 Agency for Health Technology Assessment and Tariff System (AOTMiT), Warsaw, Poland

12 Faculty of Public Health, Sofia University, Sofia, Bulgaria

13 Pharmacy Center, Faculty of Medicine, Vilnius University, Vilnius, Lithuania 